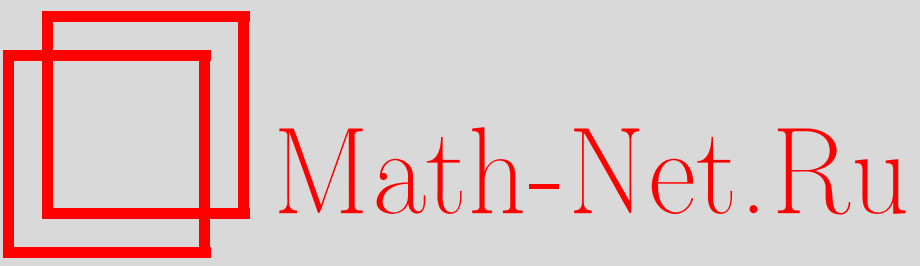

С. П. Суетин, Об асимптотике диагональных аппроксимаций Паде для гиперэллиптических функций рода $g=2$, УМH, 2003, том 58, выпуск 4, 159-160

DOI: https://doi.org/10.4213/rm654

Использование Общероссийского математического портала Math-Net.Ru подразумевает, что вы прочитали и согласны с пользовательским соглашением

http://www.mathnet.ru/rus/agreement

Параметры загрузки:

IP: 18.207 .199 .55

26 апреля 2023 г., 16:41:11 


\title{
ОБ АСИМПТОТИКЕ ДИАГОНАЛЬНЫХ АППРОКСИМАЦИЙ ПАДЕ ДЛЯ ГИПЕРЭЛЛИПТИЧЕСКИХ ФУНКЦИЙ РОДА $g=2$
}

\author{
С. П. Суетин
}

1. Недавно Д. Любинский [1] и В. И. Буслаев [2], [3] опровергли гипотезу Бейкера-ГаммеляУиллса ("Паде-гипотезу") [4], [5]. Контрпример Любинского основан на классической непрерьвной дроби Роджерса-Рамануджана, а соответствующая функция мероморфна в единичном круге $U:|z|<1$. Найденный Буслаевым контрпример особенно нагляден: функция Буслаева - гиперэллиптическая функция рода $g=2$, голоморфная в $U$. В обоих случаях соответствующая последовательность диагональных аппроксимаций Паде $\left\{f_{n}\right\}$ не содержит подпоследовательности, которая равномерно сходилась бы (к $f$ ) внутри $U$.

Для $f \in \mathscr{H}(0)$ диагональная аппроксимация Паде $f_{n}=p_{n} / q_{n}$, где $p_{n}, q_{n} \in \mathbb{C}[z], \operatorname{deg} p_{n}$, $\operatorname{deg} q_{n} \leqslant n, q_{n} \not \equiv 0$ и $\left(q_{n} f-p_{n}\right)(z)=O\left(z^{2 n+1}\right), z \rightarrow 0$. В соответствии с общей теоремой Шталя [6] о сходимости аппроксимаций Паде, имеем: если $f \in \mathscr{H}(0)$ - алгебраическая функиия, то последовательность $\left\{f_{n}\right\}$ сходится $\mathcal{K} f$ по емкости внутри области $G=\overline{\mathbb{C}} \backslash \Sigma$, где "симметричный" компакт $\Sigma$ состоит из конечного числа кусочно-аналитических дуг, а $f$ продолжается в $G$ как мероморфная функция. Замечено [7], что препятствием к равномерной сходимости последовательности $\left\{f_{n}\right\}$ является лишь конечное число полюсов $f_{n}$ с "неопределенным" поведением. Для эллиптических функций в [8] было показано, что в общем положении такие "блуждающие" полюсы всюду плотны в $\overline{\mathbb{C}}$.

В контрпримерах Любинского и Буслаева именно наличие блуждающих полюсов играет ключевую роль: для некоторой области $\Omega \Subset U$ функция $f \in \mathscr{H}(\Omega)$, но при всех $n \in \mathbb{N}$ в $\Omega$ имеется полюс $f_{n}$ (см. также [9]).

Шталем [10] было показано, что для гиперэллиптических функций, риманова поверхность которых находится в общем положении, Паде-гипотеза верна. В [11] (см. также [12], [13]) был предложен новьй метод, основанньй на краевой задаче Римана, которьй позволил получить естественное обобщение резултата Шталя. Здесь мы применяем этот метод для анализа примера Буслаева.

2. Функция Буслаева имеет вид $f(z)=\left(p_{3}(z)+\sqrt{h_{6}(z)}\right) / q_{3}(z)$, где $p_{3}(z)=-27+6 z^{2}+$ $3(9+\varepsilon) z^{3}, q_{3}(z)=2 z\left(9+9 z+(9+\varepsilon) z^{2}\right), h_{6}(z)=81\left(3-(3+\varepsilon) z^{3}\right)^{2}+4 z^{6}, \varepsilon=-1 / 2+i \sqrt{3} / 2$, $\varepsilon^{3}=1$. Ветвь корня выбрана так, что $f \in \mathscr{H}(0)$, при этом $f(0)=0$. Функция $f$ определена на гиперэллиптической римановой поверхности рода $g=2$. Полином $q_{3}(z)=c z(z-a)(z-b)$, где $|a|>1,|b|<1$. Функция $f$ голоморфна в точке $z=b$ и имеет простой полюс в точке $z=a$. "Симметричньй" компакт $\Sigma$, соответствующий функции $\sqrt{h_{6}}$, состоит из 3 непересекающихся дуг: $\Sigma=\Sigma_{1} \sqcup \Sigma_{2} \sqcup \Sigma_{3}$.

Нам удобно сделать замену переменных $\zeta=1 / z$ и перейти к функции $F(\zeta)=f(z), F \in$ $\mathscr{H}(\infty)$. Компакт $\Sigma$ перейдет в компакт $S=S_{1} \sqcup S_{2} \sqcup S_{3}$. Будем считать, что риманова поверхность $\mathfrak{R}: w^{2}=h_{6}(1 / \zeta)$ реализована как двулистное накрытие над $\overline{\mathbb{C}}$, разветвленное в концах дуг $S_{j}$. Первый (открытый) лист $D^{(1)}$ будем отождествлять с “физической” областью $D=\overline{\mathbb{C}} \backslash S$. Переходна второй лист $D^{(2)}$ осуществляется по "верхнему" и нижнему “берегам" ориентированных кривых $S_{j}$, которым соответствуют на $\mathfrak{R}$ замкнутые циклы $\Gamma_{j}$. Функция $F$ голоморфина при $|\zeta|>1$, мероморфна на $\mathfrak{R}$ и имеет простые полюсы в точках $\boldsymbol{\zeta}=\alpha^{(1)}, \beta^{(2)}, \infty^{(2)}$, где $\boldsymbol{\zeta}=\zeta^{(1)}$ или $\zeta^{(2)}, \alpha=1 / a, \beta=1 / b$. Диагональные аппроксимации Паде инвариантны относительно сделанной замены: $F_{n}(\zeta)=f_{n}(z), F_{n}(\zeta)=P_{n}(\zeta) / Q_{n}(\zeta)$, где $P_{n}$ и $Q_{n}-$ полиномы степени $\leqslant n$ и $R_{n}(\zeta):=\left(Q_{n} F-P_{n}\right)(\zeta)=O\left(1 / \zeta^{n+1}\right), \zeta \rightarrow \infty$. Функция остатка $R_{n}$ мероморфина на $\mathfrak{R}$ и имеет дивизор $\left(R_{n}\right)=(n+1) \infty^{(1)}+\zeta_{1}(n)+\zeta_{2}(n)-(n+1) \infty^{(2)}-\alpha^{(1)}-\beta^{(2)}$, где $\boldsymbol{\zeta}_{j}(n)$ - свободные нули $R_{n}$ (точки, в которых $F_{n}$ интерполирует $F$ ). Резултат Буслаева допускает

Работа выполнена при поддержке Российского фонда фундаментальных исследований (грант № 02-01-00564) и Программы поддержки ведущих научных школ России (грант НШ-1551.2003.1). 
следующую интерпретацию: $\mathbb{N}=\Lambda_{0} \sqcup \Lambda_{1} \sqcup \Lambda_{2}$ так, что: $\boldsymbol{\zeta}_{1}=\alpha^{(1)}, \boldsymbol{\zeta}_{2}=\beta^{(2)}$ при $n \in \Lambda_{0}$; $\boldsymbol{\zeta}_{1}=\varepsilon \alpha^{(1)}, \zeta_{2}=\varepsilon \beta^{(2)}$ при $n \in \Lambda_{1} ; \boldsymbol{\zeta}_{1}=\varepsilon^{2} \alpha^{(1)}, \zeta_{2}=\varepsilon^{2} \beta^{(2)}$ при $n \in \Lambda_{2}$. Таким образом, $F_{n}\left(\zeta_{1}\right)=F\left(\zeta_{1}\right)$, но $F_{n}\left(\zeta_{2}\right)=F\left(\zeta_{2}^{(2)}\right)$, т.е. в точках $\zeta_{2}(n) \in D$ аппроксимации Паде интерполируют другую ветвь функции $F$.

3. Пусть $d \Omega_{1}, d \Omega_{2}$ - нормированный относительно кривых $\Gamma_{1}, \Gamma_{2}$ базис голоморфиных абелевых дифференциалов на $\mathfrak{R}$. Из теоремы Абеля вытекает, что точки $\boldsymbol{\zeta}_{1}, \boldsymbol{\zeta}_{2}$ удовлетворяют системе уравнений

$$
\Omega_{k}\left(\boldsymbol{\zeta}_{1}\right)+\Omega_{k}\left(\boldsymbol{\zeta}_{2}\right) \equiv \Omega_{k}\left(\alpha^{(1)}\right)+\Omega_{k}\left(\beta^{(2)}\right)+2 \Omega_{k}\left(\infty^{(2)}\right)+2 n \Omega_{k}\left(\infty^{(2)}\right), \quad k=1,2,
$$

где равенство “三” понимается по модулю периодов дифференциалов $d \Omega_{k}$, а многозначные функции $\Omega_{k}(\zeta)$ нормированы условием $\Omega_{k}\left(\infty^{(1)}\right) \equiv-\Omega_{k}\left(\infty^{(2)}\right)$. Так как $\alpha \neq \beta$, то дивизор $d(n)=$ $\boldsymbol{\zeta}_{1}(n)+\boldsymbol{\zeta}_{2}(n)$ неспециальный при каждом $n \in \mathbb{N}$ и (1) имеет единственное решение. Система (1) при заданной правой части представляет собой проблему обращения Якоби. Рассмотрим проблему обращения Якоби

$$
\Omega_{k}\left(\widetilde{\boldsymbol{\zeta}}_{1}\right)+\Omega_{k}\left(\widetilde{\boldsymbol{\zeta}}_{2}\right) \equiv \frac{i}{\pi} \int_{S} \log \rho(\zeta) d \Omega_{k}^{+}(\zeta)+2 n \Omega_{k}\left(\infty^{(2)}\right), \quad k=1,2,
$$

где $\rho \neq 0$. Можно подобрать функцию $\rho$ так, чтобы

$$
\frac{i}{\pi} \int_{S} \log \rho(\zeta) d \Omega_{k}^{+}(\zeta) \equiv \Omega_{k}\left(\alpha^{(1)}\right)+\Omega_{k}\left(\beta^{(2)}\right)+2 \Omega_{k}\left(\infty^{(2)}\right), \quad k=1,2
$$

(задав, например, $\rho$ равной постоянной на каждой дуге $S_{j}$ ). Тогда правые части $(1)$ и $(2)$ совпадают при всех $n \in \mathbb{N}$. Дивизор $d(n)$ неспециален, поэтому $\widetilde{\boldsymbol{\zeta}}_{1}=\boldsymbol{\zeta}_{1}, \widetilde{\boldsymbol{\zeta}}_{2}=\boldsymbol{\zeta}_{2}$ при всех $n$. Так как $\left\{\zeta_{1}(n), \zeta_{2}(n)\right\}_{n \in \mathbb{N}} \Subset D$, то при каждом $n \in \mathbb{N}$ существует кусочно-мероморфная на $\Re \backslash \Gamma$, $\Gamma=\Gamma_{1} \cup \Gamma_{2} \cup \Gamma_{3}$, функция $\Psi_{n}(\zeta)$ такая, что дивизор $\left(\Psi_{n}\right)=\zeta_{1}+\zeta_{2}+(n-2) \infty^{(2)}-n \infty^{(1)}$ и вьполняется краевое условие $\rho(\zeta) \Psi_{n}^{+}(\boldsymbol{\zeta})=\Psi_{n}^{-}(\boldsymbol{\zeta}), \boldsymbol{\zeta} \in \Gamma$ (здесь предполагается, что $\rho$ голоморфна на $S)[11]-[13]$. При этом для функции $\widehat{\rho}(\zeta)=\frac{1}{\pi i} \int_{S} \frac{\rho(t)}{w^{+}(t)} \frac{d t}{t-\zeta}$ знаменатели $Q_{n}(\zeta ; \widehat{\rho})$ ее диагоналњных аппроксимаций Паде при подходящей нормировке имеют следующую асимптотику: $Q_{n}(\zeta ; \widehat{\rho})=\Psi_{n}\left(\zeta^{(1)}\right)(1+o(1)), n \rightarrow \infty$. Тем самым, асимптотическое поведение блуждающих полюсов диагоналњных аппроксимаций Паде голоморфной в $D=\overline{\mathbb{C}} \backslash S$ функции $\widehat{\rho}$ и мероморфной в $D$ функции Буслаева совпадают. Следовательно, справедливо следующее

ПредЛОжЕнИЕ. Любая голоморфная в $D$ функция $\widehat{\rho}$, для которой $\rho \neq 0$ голоморфна на $S$ и удовлетворяет условиям (3), является контрпримером к Паде-гипотезе.

\section{СПИСОК ЛИТЕРАТУРЫ}

[1] D. Lubinsky // Ann. of Math. 2003. V. 157. №4. P. 847-889. [2] V. I. Buslaev // East J. Approx. 2001. V. 7. №4. Р. 515-517. [3] В.И. Буслаев // Матем. сб. 2002. Т. 193. №6. С. 25-38. [4] Дж. Бейкер, П. Грейвс-Моррис. Аппроксимации Паде. М.: Мир, 1986. [5] H. Stahl // Constr. Approx. 1997. V. 13. № 2. P. 287-292. [6] H. Stahl // Constr. Approx. 1986. V. 2. № 3. P. 225-240; 241-251. [7] J. Nuttall // J. Approx. Theory. 1984. V. 42. № 4. P. 299-386. [8] S. Dumas. Sur le développement des fonctions elliptiques en fractions continues. Thesis. Zürich, 1908. [9] G. A. Baker // Elsevier preprint (to appear). [10] H. Stahl // Ann. Fac. Sci. Toulouse Math. (6). 1996. Spec. Iss. P. 121-193. [11] С. П. Суетин // Матем. сб. 2000. Т. 191. №9. С. 81-114. [12] С.П. Суетин // УМН. 2002. Т. 57. №1. С. 45-142. [13] С. П. Суетин // Матем. сб. 2002. Т. 193. № 12. С. 81-114. 